\title{
Effets de la syphilis infectieuse chez les patientes enceintes et facteurs maternels associés au diagnostic de syphilis congénitale, Alberta, 2017 à 2020
}

\author{
Jennifer Gratrix ${ }^{1}$, Jennifer Karwacki ${ }^{1}$, Lynn Eagle ${ }^{1}$, Lindsay Rathjen ${ }^{1}$, Ameeta Singh ${ }^{2}$, Angel Chu ${ }^{1,3}$, \\ Petra Smyczek ${ }^{1,2 \star}$
}

\section{Résumé}

Contexte : La syphilis congénitale représente un problème de santé publique important et exige un diagnostic et un traitement précoces pour améliorer les effets des nourrissons. Cette étude vise à décrire les effets en santé publique des cas de syphilis infectieuse chez les patientes enceintes et les facteurs associés à un diagnostic de syphilis congénitale chez leur nourrisson.

Méthodes : Nous avons effectué un examen rétrospectif des caractéristiques démographiques et cliniques des cas de syphilis infectieuse diagnostiqués pendant la grossesse et des effets pour les nourrissons en Alberta de 2017 à 2020 à partir de la base de données provinciale sur les maladies transmissibles. Un traitement adéquat à la mère a été défini comme la réception d'au moins une dose de pénicilline G-LA benzathine avec 2,4 millions d'unités de MI au moins 28 jours avant l'accouchement. Une analyse à une et plusieurs variables a été effectuée pour déterminer les facteurs associés au diagnostic de syphilis congénitale à l'aide de la version 25 de SPSS.

Résultats : Au total, 374 cas de syphilis infectieuse ont été diagnostiqués pendant la grossesse, et deux patientes ont reçu un diagnostic deux fois au cours d'une même grossesse. La majorité $(79,1 \% ; n=296)$ des femmes ont eu un accouchement vivant, et une minorité a eu un avortement thérapeutique $(9,4 \% ; n=35)$, une mortinaissance $(7,5 \% ; n=28)$ et un avortement spontané $(4,0 \% ; n=15)$. Les dossiers de nourrissons $(n=265)$ étaient disponibles aux fins d'examen ( $n=117$ cas de syphilis congénitale et 148 cas sans syphilis congénitale). Les corrélations avec les cas de syphilis congénitale étaient le temps de dépistage au troisième trimestre (rapport de cotes rajusté [RCA] 8,4, intervalle de confiance [IC] à $95 \%, 2,9-24,6$ ) et moins de 28 jours avant l'accouchement (RCA 8,1, 1,4-47,8 [par rapport au premier et au deuxième trimestre]) et le traitement inadéquat (RCA 86,1, IC, 15,9-466,5). Parmi les cas de syphilis congénitale, $23,1 \%(n=27)$ étaient des mortinaissances comparativement à un $(0,7 \%)$ cas de mortinaissance chez les nourrissons sans syphilis congénitale $(p<0,001)$.

Conclusion : Le dépistage et le traitement précoces de la syphilis pendant la grossesse sont essentiels pour prévenir les effets néfastes chez les nourrissons.

Citation proposée : Gratrix J, Karwacki J, Eagle L, Rathjen L, Singh AE, Chu A, Smyczek P. Résultats de la syphilis infectieuse chez les patientes enceintes et facteurs maternels associés au diagnostic de syphilis congénitale, Alberta, 2017 à 2020. Relevé des maladies transmissibles au Canada 2022;48(2/3):68-75.

https://doi.org/10.14745/ccdr.v48i23a02f

Mots-clés : syphilis, infections transmissibles sexuellement, syphilis congénitale, dépistage prénatal
Cette oeuvre est mise à la disposition selon les termes de la licence internationale Creative Commons Attribution 4.0

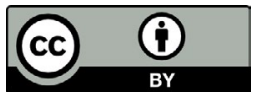

Affiliations

${ }^{1}$ Service des infections transmissibles sexuellement, Alberta Health Services, Edmonton, $A B$

2 Département de médecine, Université de l'Alberta, Edmonton, $A B$

${ }^{3}$ Département de médecine, École de médecine Cumming, Calgary, AB

*Correspondance : petra.smyczek@ albertahealthservices.ca 


\section{Introduction}

La syphilis congénitale est un problème de santé publique à l'échelle mondiale et reflète l'incidence de la syphilis infectieuse chez les femmes hétérosexuelles (1). Si elle n'est pas traitée, la syphilis pendant la grossesse peut avoir une grave incidence sur l'issue de la grossesse, entraînant même un avortement spontané, une mortinaissance, une mort périnatale et des séquelles graves chez les nourrissons infectés.

L'Organisation mondiale de la Santé a déterminé des cibles mondiales pour 2030 afin de réduire l'incidence de la syphilis de $90 \%$ et de réduire le nombre de cas de syphilis congénitale à 50 ou moins pour 100000 naissances vivantes dans $80 \%$ des pays (2). En 2016, le taux mondial estimé de cas de syphilis congénitale était de 473

(étendue $=385-561$ ) pour 100000 naissances vivantes et de 661000 (étendue $=538000-784000$ ) cas de syphilis congénitale totaux (3).

Une éclosion provinciale de syphilis a été déclarée en Alberta en 2016, après que les taux d'infection ont doublé, passant de 3,9/100 000 habitants en 2014 à 8,8/100 000 habitants en 2015 et ont grimpé à 56,7/100 000 habitants en 2020. Pendant cette période, le taux chez les femmes a été multiplié par 90 , passant de 0,6/100 000 habitantes en 2014 à 49,6/100 000 habitantes en 2020 (4). La proportion de cas pendant les grossesses est passée de $0 \%$ en 2014 à $15 \%$ en 2020 (données non publiées). De 2012 à 2016, un seul cas de syphilis congénitale a été signalé, comparativement à 121 cas de syphilis congénitale signalés de 2017 à 2020, avec 45 et 56 cas diagnostiqués en 2019 et en 2020, respectivement (4). Pendant cette période, 28 nourrissons étaient mort-nés.

L'objectif de cette étude est de décrire l'épidémiologie des cas de syphilis infectieuse diagnostiqués pendant la grossesse et de faire état des effets de la grossesse et pour le nouveau-né.

\section{Méthodes}

Nous avons effectué un examen rétrospectif sur les caractéristiques démographiques et cliniques des cas de syphilis infectieuse diagnostiqués pendant la grossesse et des effets de grossesse et néonatals qui en découlent en Alberta (population actuelle de 4,3 millions de personnes) de 2017 à 2020. En Alberta, le dépistage prénatal de la syphilis est recommandé pour tous au cours du premier trimestre, au moment de l'accouchement et tout au long de la grossesse pour les personnes à risque continus $(5,6)$. Tous les cas de syphilis doivent être déclarés par les laboratoires et les cliniciens au programme provincial des infections transmissibles sexuellement (ITS), qui est responsable d'assurer un traitement adéquat et de prévenir les partenaires de tous les cas confirmés. Les infirmières responsables de la notification des partenaires (INP) agissent à titre de gestionnaires de cas pour les patients en collaborant avec le patient, les professionnels de la santé responsables des tests et du traitement et le médecin-conseil en ITS pour faciliter l'évaluation, le traitement et le suivi appropriés des patients et de leurs partenaires sexuels. Toutes les patientes enceintes sont évaluées au moyen d'entrevues menées par des professionnels de la santé ou des INP. Pour les patientes enceintes, les INP coordonnent également les soins avec le site d'accouchement afin d'assurer la sérologie au moment de l'accouchement et le suivi approprié des nourrissons.

Des conseillers médicaux spécialisés en infections transmissibles sexuellement effectuent l'évaluation du stade de la syphilis de la mère conformément aux définitions de cas provinciales (7) et ils examinent les nourrissons pour déterminer s'il y a des diagnostics possibles de syphilis congénitale.

Les données anonymisées ont été extraites de la base de données provinciale sur les maladies transmissibles le 25 juin 2021; les mères et les nourrissons ont été couplés au moyen de numéros d'enregistrement uniques. Les variables extraites pour chaque cas de syphilis comprenaient des facteurs démographiques, comportementaux et cliniques. L'origine ethnique, les antécédents de rapports sexuels transactionnels, I'usage de drogues par injection (UDI), les rapports sexuels avec une personne qui consomme des drogues par injection ont été autodéclarés pendant les entrevues avec les INP dans le cadre des exigences de suivi et de déclaration de routine en matière de santé publique. Une recherche de symptômes pour les plaies, les éruptions cutanées, les lésions et les signes neurologiques a été effectuée par la patiente ou par le professionnel de la santé. L'intervention correctionnelle incluait tout cas où la patiente a d'abord été diagnostiquée ou traitée dans un établissement correctionnel. L'Alberta est divisée en cinq zones géographiques qui ont été attribuées en fonction du code postal de la patiente. La proximité en matière de soins de santé a été définie en fonction de la densité de la population et des temps de déplacement vers les services de santé : régions métropolitaines et urbaines (municipalités ayant une densité de population d'au moins 20000 habitants par $\mathrm{km}^{2}$ et collectivités adjacentes dotées d'un hôpital régional ou tertiaire); régions rurales (densité de la population entre 100 et 10000 habitants par $\mathrm{km}^{2}$ ); et régions rurales éloignées (densité de la population inférieure à 100 habitants par $\mathrm{km}^{2}$ et distance supérieure à $200 \mathrm{~km}$ pour un centre régional). Les contacts avec la santé publique ont été définis comme étant des patientes contactées par les INP par rapport aux patientes que les INP n'ont pas pu contacter. Le temps de dépistage était la différence entre la date de l'accouchement et la date de collecte du premier échantillon positif, stratifié en trois catégories : premier et deuxième trimestres (au moins 91 jours); troisième trimestre (28 à 90 jours), et moins de 28 jours avant l'accouchement, puisque ce délai était insuffisant pour permettre un traitement adéquat. Un traitement 
adéquat à la mère a été défini comme la réception d'au moins une dose de pénicilline G-LA benzathine avec 2,4 millions d'unités IM ou de pénicilline G cristalline aqueuse avec 18 à 24 millions d'unités par jour pendant au moins 10 jours et plus de 28 jours avant l'accouchement. La durée du traitement a été calculée à partir de la date du premier résultat positif du test jusqu'à la dose initiale du médicament. Pour ce qui est des effets à la naissance, la mortinaissance a été définie comme la mort fœtale après 20 semaines de gestation, et l'avortement spontané était une mort fœtale à 20 semaines de gestation ou avant.

Des analyses descriptives ont été effectuées pour les cas d'UDI, les rapports sexuels avec une personne qui consomme des drogues par injection et les rapports sexuels transactionnels; toutefois, en raison de fortes proportions de données manquantes (25\% à $31 \%$ ), ces variables ont été exclues d'une analyse supplémentaire. Afin de déterminer les facteurs associés à la syphilis congénitale, des analyses ont été effectuées en comparant les cas de syphilis congénitale et les cas sans syphilis congénitale. Des analyses à une variable ont été effectuées à l'aide du test exact du khi carré ou de la méthode exacte de Fisher pour les variables de catégories. Nous avons utilisé le test de Mann-Whitney pour les variables continues, à l'exclusion des données manquantes. Pour déterminer les facteurs indépendants associés à la syphilis congénitale, nous avons effectué une régression logistique à plusieurs variables. Toutes les variables significatives à $p \leq 0,10$ au niveau à une variable ont été évaluées pour la multicollinéarité. On a constaté une forte corrélation entre le nombre de jours de traitement et le traitement adéquat, de sorte que le nombre de jours de traitement n'a pas été inclus dans l'élaboration du modèle. Les modèles ont été conçus à I'aide de toutes les variables importantes, et nous avons constaté que l'approche progressive avait la meilleure cote d'adéquation d'Hosmer-Lemeshow. Les analyses ont été effectuées à l'aide de IBM SPSS Statistics 19 (IBM, Armonk, New York, États-Unis).

\section{Résultats}

Au total, 374 cas de syphilis infectieuse ont été diagnostiqués pendant la grossesse entre le 1 er janvier 2017 et le 31 décembre 2020, et deux patientes ont été réinfectées au cours de la même grossesse. La majorité des cas se sont déclaré chez des femmes autochtones résidant dans la zone d'Edmonton, provenant d'une région métropolitaine ou urbaine ayant été diagnostiquée avec la syphilis au stade latent précoce. Elles avaient été contactée par une INP et avaient déjà eu une naissance vivante. Tous les cas étaient négatifs pour le $\mathrm{VIH}$. Une proportion importante de femmes a déclaré une UDI $(21,0 \% ; n=58 / 276)$, de rapports sexuels avec une personne qui consomme des drogues par injection $(18,1 \% ; n=47 / 259)$ et des rapports sexuels transactionnels $(11,0 \% ; n=31 / 281)$; cependant, ces variables présentaient des proportions élevées de données manquantes (tableau 1).
Tableau 1 : Caractéristiques maternelles des cas de syphilis infectieuse chez les patientes enceintes en Alberta, 2017 à $2020(n=374)$

\begin{tabular}{|c|c|c|}
\hline Caractéristiques & $\mathbf{N}=374$ & $\%^{a}$ \\
\hline
\end{tabular}

Âge médian au moment du diagnostic (années, El) 26 $2 \cdot-30$ Ethnicité

\begin{tabular}{|l|r|r|}
\hline Caucasienne & 58 & 15,5 \\
\hline Premières Nations & 228 & 61,0 \\
\hline Métis & 36 & 9,6 \\
\hline Autre & 6 & 1,6 \\
\hline Inconnu/manquant & 46 & 12,3 \\
\hline Établissement correctionnel & & \\
\hline Non & 342 & 91,4 \\
\hline Oui & 32 & 8,6 \\
\hline
\end{tabular}

Zone géographique

\begin{tabular}{|l|r|r|}
\hline Sud & 2 & 0,5 \\
\hline Calgary & 16 & 4,3 \\
\hline Centre & 30 & 8,0 \\
\hline Edmonton & 217 & 58,0 \\
\hline Nord & 109 & 29,1
\end{tabular}

Proximité médicale

\begin{tabular}{|l|r|r|}
\hline Région métropolitaine/urbaine & 229 & 61,2 \\
\hline Région rurale & 103 & 27,5 \\
\hline Région rurale éloignée & 42 & 11,2 \\
\hline Année de diagnostic & & \\
\hline
\end{tabular}

\begin{tabular}{|r|r|r|}
\hline 2017 & 15 & 4,0 \\
\hline 2018 & 66 & 17,6 \\
\hline 2019 & 145 & 38,8 \\
\hline 2020 & 148 & 39,6 \\
\hline
\end{tabular}

Contact avec la santé publique

\begin{tabular}{|l|r|r|}
\hline Cliente contactée & 325 & 86,9 \\
\hline Impossible de la joindre & 49 & 13,1 \\
\hline
\end{tabular}

\begin{tabular}{|l|r|r|}
\hline Stade de la syphilis & \multicolumn{3}{|c|}{} \\
\hline Primaire & 114 & 30,5 \\
\hline
\end{tabular}

\begin{tabular}{|l|r|r|}
\hline Primaire & 114 & 30,5 \\
\hline Secondaire & 21 & 5,6 \\
\hline Latence précoce & 238 & 63,6 \\
\hline Neurosyphilis précoce & 1 & 0,3 \\
\hline Jours médians de traitement ${ }^{\mathrm{b}}$ (EI) & 6 & $3-13$ \\
\hline Issue de la naissance & & \\
\hline Naissance vivante & 296 & 79,1 \\
\hline Mortinaissance & 28 & 7,5 \\
\hline Avortement spontané & 15 & 4,0 \\
\hline Avortement thérapeutique & 35 & 9,4 \\
\hline
\end{tabular}

Abréviation : El écart interquartile

a Ce tableau représente 374 cas de syphilis infectieuse parmi 372 grossesses : deux patientes ont été réinfectées au cours de la même grossesse

${ }^{b}$ Cinq cas n'avaient pas de dossier de traitement au moment du diagnostic

Sur les 324 cas de naissance vivante ou de mortinaissance, des dossiers sur les nourrissons étaient disponibles pour tous les cas, sauf trois. Sept paires de jumeaux sont nées, ce qui donne un total de 328 dossiers de nourrissons à examiner. Près du 
tiers $(35,7 \% ; n=117)$ des nourrissons ont reçu un diagnostic de syphilis congénitale, $45,1 \%(n=148)$ des nourrissons ne répondaient pas aux définitions des cas de syphilis congénitale (sans syphilis congénitale) et il n'y a pas de détermination de stade pour 19,2\% ( $n=63)$ des nourrissons qui font l'objet d'un suivi continu. Les caractéristiques maternelles associées à la syphilis congénitale dans l'analyse à une variable étaient l'absence de contact avec l'INP, le fait d'avoir deux partenaires sexuels ou plus au cours de la dernière année, le temps de dépistage au troisième trimestre ou moins de 28 jours avant l'accouchement, et le fait de ne pas recevoir un traitement adéquat (tableau 2 et tableau 3). Sur un total de 265 cas, un tiers $(35,8 \% ; n=95)$ des patientes ont été examinées moins d'un mois avant l'accouchement, et bon nombre d'entre elles (25,3\%; $n=67)$ l'ont été dans les deux jours suivant I'accouchement. La majorité $(61,1 \% ; n=162)$ des patients ont reçu un traitement adéquat pour la prévention de la syphilis congénitale; 161 patientes ont reçu de la pénicilline G-LA benzathine avec 2,4 millions d'unités d'IM au moins 28 jours avant l'accouchement et une patiente a reçu de la pénicilline $\mathrm{G}$ cristalline aqueuse avec 24 millions d'unités par jour pendant 14 jours. Les $39,2 \%$ restants $(n=104)$ des patientes $n^{\prime}$ ont pas été traitées adéquatement. Le quart $(24,5 \% ; n=65)$ des patientes a été traité avec au moins une dose unique de pénicilline G-LA benzathine avec 2,4 millions d'unités d'IM au moment de l'accouchement ou après, 11,3\% $(n=30)$ ont été traitées de 1 à 27 jours avant l'accouchement, deux patientes $(0,8 \%)$ ont reçu de la doxycycline $100 \mathrm{mg}$ deux fois par jour pendant 14 jours après l'accouchement, et une patiente a reçu un traitement insuffisant avec de la pénicilline $\mathrm{G}$ cristalline aqueuse avec 5 millions d'unités par voie intraveineuse en une seule dose après l'accouchement. Cinq autres cas (1,9\%) n'avaient aucun dossier de traitement au moment de l'analyse. Les corrélats qui sont demeurés indépendamment associés à la syphilis congénitale après une analyse à plusieurs variables étaient le temps de dépistage au troisième trimestre (RCA 8,4, IC à $95 \%, 2,9-24,6)$ et moins de 28 jours avant l'accouchement (RCA 8.1, 1,4-47,8 [par rapport au premier et deuxième trimestre]) et traitement inadéquat (RCA 86,1, IC, 15,9-466,5). Parmi les cas de syphilis congénitale, $23,1 \%(n=27 / 117)$ étaient des mortinaissances comparativement à un cas $(0,7 \%)$ de mortinaissance chez les nourrissons sans syphilis congénitale $(p<0,001)$. Malgré le diagnostic au cours du premier et deuxième trimestre de neuf cas de syphilis congénitale, quatre cas ont été traités moins de 28 jours avant l'accouchement. Sur les 237 naissances vivantes, tous les cas de syphilis congénitale ont reçu un traitement. Trente $(20,4 \%)$ des cas sans syphilis congénitale ont également reçu un traitement; ces cas ne répondaient pas à la définition de cas de l'Alberta pour la syphilis congénitale (8), mais ils ont été traités au moment de l'accouchement en fonction du jugement clinique et des antécédents maternels connus au moment de l'admission après évaluation par des spécialistes des maladies infectieuses pédiatriques.

\section{Tableau 2 : Analyse à une variable des caractéristiques de la mère, associées aux effets du nourrisson pour les cas de syphilis infectieuse chez les patientes enceintes en Alberta, 2017 à $2020(N=265)$}

\begin{tabular}{|c|c|c|c|c|c|c|c|c|c|}
\hline \multirow[t]{2}{*}{ Caractéristiques maternelles } & \multicolumn{2}{|c|}{$\begin{array}{c}\text { Infection } \\
\text { congénitale }\end{array}$} & \multicolumn{2}{|c|}{ Sans infection } & \multicolumn{2}{|c|}{ Total } & \multirow[t]{2}{*}{ valeur $p$} & \multicolumn{2}{|c|}{$\begin{array}{l}\text { Rapport de cotes } \\
\text { non rajusté }\end{array}$} \\
\hline & $(n=117)$ & $\%$ & $(n=148)$ & $\%$ & $(N=265)$ & $\%$ & & Taux & IC à $95 \%$ \\
\hline $\begin{array}{l}\text { Âge médian au moment du diagnostic } \\
\text { (années, El) }\end{array}$ & 27 & $23-31$ & 26 & $21-30$ & 26 & $22-31$ & 0,18 & 1,0 & $1,0-1,1$ \\
\hline \multicolumn{10}{|l|}{ Ethnicité } \\
\hline Caucasienne & 17 & 14,5 & 24 & 16,2 & 41 & 15,5 & 0,37 & Réf. & Réf. \\
\hline Premières Nations & 71 & 60,7 & 96 & 64,9 & 167 & 63,0 & - & 1,0 & $0,5-2,1$ \\
\hline Métis & 13 & 11,1 & 7 & 4,7 & 20 & 7,5 & - & 2,6 & $0,9-8,0$ \\
\hline Autre & 1 & 0,9 & 3 & 2,0 & 4 & 1,5 & - & 0,5 & $0,1-4,9$ \\
\hline Inconnu/manquant & 15 & 12,8 & 18 & 12,2 & 33 & 12,5 & - & 1,2 & $0,5-3,0$ \\
\hline \multicolumn{10}{|l|}{ Établissement correctionnel } \\
\hline Non & 107 & 91,5 & 133 & 89,9 & 240 & 90,6 & 0,66 & Réf. & Réf. \\
\hline Oui & 10 & 8,5 & 15 & 10,1 & 25 & 9,4 & - & 0,8 & $0,4-1,9$ \\
\hline \multicolumn{10}{|l|}{ Zone géographique } \\
\hline Calgary & 4 & 3,4 & 5 & 3,4 & 9 & 3,4 & 0,05 & Réf. & Réf. \\
\hline Centre & 16 & 13,7 & 6 & 4,1 & 22 & 8,3 & - & 3,3 & $0,7-16,7$ \\
\hline Edmonton & 63 & 53,8 & 90 & 60,8 & 153 & 57,7 & - & 0,9 & $0,2-3,4$ \\
\hline Nord & 34 & 29,1 & 47 & 31,8 & 81 & 30,6 & - & 0,9 & $0,2-3,6$ \\
\hline \multicolumn{10}{|l|}{ Proximité médicale } \\
\hline Région métropolitaine/urbaine & 65 & 55,6 & 93 & 62,8 & 158 & 59,6 & 0,09 & Réf. & Réf. \\
\hline Région rurale & 39 & 33,3 & 32 & 21,6 & 71 & 26,8 & - & 1,7 & $1,0-3,1$ \\
\hline Région rurale éloignée & 13 & 11,1 & 23 & 15,5 & 36 & 13,6 & - & 0,8 & $0,4-1,7$ \\
\hline
\end{tabular}


Tableau 2 : Analyse à une variable des caractéristiques de la mère, associées aux effets du nourrisson pour les cas de syphilis infectieuse chez les patientes enceintes en Alberta, 2017 à 2020 ( $N=265)$ (suite)

\begin{tabular}{|c|c|c|c|c|c|c|c|c|c|}
\hline \multirow[t]{2}{*}{ Caractéristiques maternelles } & \multicolumn{2}{|c|}{$\begin{array}{c}\text { Infection } \\
\text { congénitale }\end{array}$} & \multicolumn{2}{|c|}{ Sans infection } & \multicolumn{2}{|c|}{ Total } & \multirow[t]{2}{*}{ valeur $p$} & \multicolumn{2}{|c|}{$\begin{array}{l}\text { Rapport de cotes } \\
\text { non rajusté }\end{array}$} \\
\hline & $(n=117)$ & $\%$ & $(n=148)$ & $\%$ & $(\mathbf{N}=265)$ & $\%$ & & Taux & IC à $95 \%$ \\
\hline \multicolumn{10}{|l|}{ Année de diagnostic } \\
\hline 2017 & 5 & 4,3 & 9 & 6,1 & 14 & 5,3 & 0,01 & 1,6 & $1,2-2,1$ \\
\hline 2018 & 16 & 13,7 & 43 & 29,1 & 59 & 22,3 & - & - & - \\
\hline 2019 & 44 & 37,6 & 52 & 35,1 & 96 & 36,2 & - & - & - \\
\hline 2020 & 52 & 44,4 & 44 & 29,7 & 96 & 36,2 & - & - & - \\
\hline \multicolumn{10}{|l|}{ Contact avec la santé publique } \\
\hline Cliente contactée & 89 & 76,1 & 136 & 91,9 & 225 & 84,9 & $<0,001$ & Réf. & Réf. \\
\hline Impossible de la joindre & 28 & 23,9 & 12 & 8,1 & 40 & 15,1 & - & 3,6 & $1,7-7,4$ \\
\hline \multicolumn{10}{|l|}{ Symptômes ${ }^{a}$} \\
\hline Non & 49 & 48,0 & 64 & 44,8 & 113 & 46,1 & 0,61 & Réf. & Réf. \\
\hline Oui & 53 & 52,0 & 79 & 55,2 & 132 & 53,9 & - & 0,9 & $0,5-1,5$ \\
\hline \multicolumn{10}{|c|}{ Nombre de partenaires au cours des 12 derniers mois } \\
\hline Moins de 2 & 21 & 17,9 & 10 & 6,8 & 31 & 11,7 & 0,005 & Réf. & Réf. \\
\hline Au moins 2 & 96 & 82,1 & 138 & 93,2 & 234 & 88,3 & - & 0,3 & $0,2-0,7$ \\
\hline \multicolumn{10}{|l|}{ Stade de la syphilis de la mère } \\
\hline Primaire & 34 & 29,1 & 43 & 29,1 & 77 & 29,1 & 1,00 & 1,0 & $0,6-1,7$ \\
\hline Secondaire & 8 & 6,8 & 9 & 6,1 & 17 & 6,4 & - & 1,1 & $0,4-3,2$ \\
\hline Latence précoce & 75 & 64,1 & 95 & 64,2 & 170 & 64,2 & - & Réf. & Réf. \\
\hline Neurosyphilis précoce & 0 & 0 & 1 & 0,7 & 1 & 0,4 & - & - & $\begin{array}{r}\text { Analyse de } \\
\text { régression } \\
\text { exclue à } 0 \\
\text { cellule }\end{array}$ \\
\hline Jours médians avant le traitement ${ }^{b}$ & 5 & $2,0-8,5$ & 6 & $\begin{array}{l}3,0- \\
10,0\end{array}$ & 6 & $3,0-10,0$ & 0,03 & 1,0 & $0,999-1,01$ \\
\hline \multicolumn{10}{|l|}{ Moment du dépistage } \\
\hline Premier et deuxième trimestre & 9 & 7,7 & 111 & 75,0 & 120 & 45,3 & $<0,001$ & Réf. & Réf. \\
\hline Troisième trimestre & 18 & 15,4 & 32 & 21,6 & 50 & 18,9 & - & 6,9 & $2,8-16,9$ \\
\hline $\begin{array}{l}\text { Au moins } 28 \text { jours après } \\
\text { l'accouchement }\end{array}$ & 90 & 76,9 & 5 & 3,4 & 95 & 35,8 & - & 222 & $71,9-685,9$ \\
\hline \multicolumn{10}{|l|}{ Traitement adéquat } \\
\hline Non & 99 & 84,6 & 4 & 2,7 & 103 & 38,9 & $<0,001$ & 198,0 & $65,0-602,7$ \\
\hline Oui & 18 & 15,4 & 144 & 97,3 & 162 & 61,1 & - & Réf. & Réf. \\
\hline
\end{tabular}

Abréviations : $\mathrm{El}$, écart interquartile; IC, intervalle de confiance; Réf., référence; -, sans objet

a Exclut 20 cas pour lesquels il manque des données

b Exclut six cas dont la date de traitement est manquante

Tableau 3 : Analyse à plusieurs variables des caractéristiques de la mère, associées aux effets du nourrisson pour les cas de syphilis infectieuse chez les patientes enceintes en Alberta, 2017 à $2020(N=265)$

\begin{tabular}{|c|c|c|c|}
\hline $\begin{array}{l}\text { Caractéristiques } \\
\text { maternelles }\end{array}$ & RCA & IC à $95 \%$ & valeur $p$ \\
\hline \multicolumn{4}{|l|}{ Temps de dépistage } \\
\hline $\begin{array}{l}\text { Premier et deuxième } \\
\text { trimestre }\end{array}$ & Réf. & - & - \\
\hline Troisième trimestre & 8,4 & $2,9-24,6$ & $<0,001$ \\
\hline $\begin{array}{l}\text { Au moins } 28 \text { jours } \\
\text { après l'accouchement }\end{array}$ & 8,1 & $1,4-47,8$ & 0,02 \\
\hline \multicolumn{4}{|l|}{ Traitement adéquat } \\
\hline Oui & Réf. & - & - \\
\hline Non & 86,1 & $15,9-466,5$ & $<0,001$ \\
\hline
\end{tabular}

\section{Discussion}

Au Canada, les taux de syphilis infectieuse ont fortement augmenté au cours des cinq dernières années; l'augmentation de $740 \%$ des cas infectieux entre 2016 et 2020 pour les femmes est particulièrement alarmante (8). Des tendances épidémiologiques semblables ont été observées aux États-Unis, avec près de 2100 cas de syphilis congénitale en 2020 (9). Depuis le début de l'éclosion de syphilis en 2015, l'Alberta a enregistré un nombre sans précédent de cas de syphilis infectieuse chez les femmes enceintes, ce qui a entraîné des cas de syphilis congénitale et des mortinaissances. Le traitement inadéquat de la mère était le prédicteur le plus important d'un diagnostic de syphilis congénitale dans notre étude. Plusieurs facteurs ont contribué à un traitement inadéquat pendant la grossesse. Premièrement, la probabilité d'accoucher d'un nourrisson atteint de syphilis 
congénitale était huit fois plus élevée chez les patientes qui ont passé un dépistage au troisième trimestre et chez celles qui ont passé un dépistage au dernier mois de la grossesse, car le diagnostic tardif ne laisse pas suffisamment de temps pour que le traitement soit efficace. De plus, quatre des neuf cas de syphilis congénitale ayant fait l'objet d'un dépistage maternel au cours du premier et deuxième trimestre n'ont pas reçu de traitement quatre semaines avant l'accouchement en raison d'obstacles à I'accès aux soins. Nos résultats sont semblables à ceux d'une étude récente sur le traitement de la syphilis maternelle qui a montré qu'après avoir reçu un traitement au cours du premier trimestre, il n'y a eu aucun cas de syphilis congénitale néonatal chez les nourrissons des patientes enceintes. Les patientes qui ont commencé le traitement au troisième trimestre présentaient un risque accru de mortinaissance, de naissance prématurée et de faible poids à la naissance (10). Une autre étude qui examinait les déterminants associés à la syphilis congénitale et aux effets indésirables pendant la grossesse a révélé que chaque semaine de retard dans le traitement était liée à un risque accru 2,82 fois plus élevé d'effets indésirables pendant la grossesse (11).

Bien que le dépistage au cours du premier trimestre et au milieu de la gestation vise à prévenir la syphilis congénitale, l'objectif du dépistage à l'accouchement est le diagnostic et le traitement précoces des nourrissons nés de mères atteintes de syphilis infectieuse. Une analyse de rentabilité réalisée aux États-Unis en 2018 a révélé que les dépistages répétés au troisième trimestre sont supérieurs aux dépistages uniques au premier trimestre et qu'ils sont à la fois rentables et entraînent une amélioration des effets pour la mère et le nouveau-né (12). Lors de la dernière éclosion de syphilis infectieuse en Alberta, au milieu des années 2000, un dépistage universel au milieu de la grossesse $a$ été mis en place. Après un examen du programme de dépistage prénatal de l'Alberta, il a été interrompu en 2012 en raison d'une faible participation et d'une utilité limitée pour le diagnostic de nouvelles infections à la syphilis (13), même si les taux de syphilis étaient plus faibles à ce moment-là. Les lignes directrices du Canada et des Centres pour le contrôle et la prévention des maladies des États-Unis sur le dépistage de la syphilis recommandent le dépistage au premier trimestre ou lors de la première visite prénatale et le dépistage répété entre les semaines 28 à 32 de gestation, puis de nouveau au moment de l'accouchement pour les patientes dans les régions où les taux de syphilis sont élevés et pour les femmes à risque continu d'infection par la syphilis $(14,15)$. Les résultats de notre étude appuient les lignes directrices actuelles de l'Alberta sur le dépistage de la syphilis chez les patientes enceintes, qui recommandent le dépistage maternel universel au premier trimestre, au moment de l'accouchement $(5,6)$ et de nouveau pour les patientes à risque continu. La majorité des mères interrogées ont déclaré avoir eu plusieurs partenaires au cours de la dernière année, et plus de la moitié d'entre elles ont signalé des symptômes, ce qui répond aux recommandations actuelles en matière de dépistage répété pendant la grossesse. Même en respectant les recommandations provinciales actuelles en matière de dépistage du risque et en exigeant un dépistage fréquent tout au long de la grossesse, nous avons constaté qu'un nombre élevé de patientes avaient passé leur premier dépistage en fin de grossesse (un quart dans les deux jours suivant l'accouchement) et qu'il y a eu deux cas de réinfection au cours de la même grossesse. Nous croyons que des tests fréquents et des tests de dépistage répétés chez les femmes enceintes, jusqu'à une fois par mois après un résultat négatif initial de femmes qui sont à risque continu, constituent un outil important pour prévenir d'autres cas de syphilis chez les nouveau-nés. L'amélioration des connaissances générales sur la syphilis et la sensibilisation aux lignes directrices existantes en matière de dépistage chez les professionnels de la santé sont des étapes importantes pour améliorer les effets en matière de santé des femmes enceintes et de leurs nourrissons. Le dépistage en temps opportun lorsque les femmes à risque se présentent pour recevoir des soins de santé pour des causes non liées à la grossesse peut mener à un diagnostic et à un traitement plus précoces, réduisant ainsi la morbidité et la mortalité liées à la syphilis congénitale. Le recours à des tests au point de soins et au traitement de la syphilis symptomatique devrait être envisagé pour les femmes à risque de ne pas être suivies. En offrant un dépistage aux femmes dans des milieux de soins non traditionnels, comme les centres de traitement des dépendances les établissements correctionnels ou les salles d'urgence, il est possible d'accroître le dépistage chez les femmes qui autrement n'obtiendraient pas de soins prénataux. La forte proportion de cas diagnostiqués au troisième trimestre dans notre étude souligne la nécessité d'approches non traditionnelles pour le dépistage et le traitement. Le nombre de patientes qui accèdent aux services en fin de grossesse est affecté par des déterminants sociaux défavorables de la santé, notamment la pauvreté et les problèmes de santé mentale et de la dépendance. De plus, une proportion importante de patientes étaient autochtones, ce qui démontre le besoin de services culturellement adaptés. En réponse à la résurgence de la syphilis infectieuse au milieu des années 2000 en Alberta, une équipe de sensibilisation dans la zone géographique d'Edmonton a engagé des infirmières autorisées au sein de l'équipe de représentants en santé communautaire autochtone afin d'offrir des soins adaptés à la culture. Les services d'approche peuvent constituer une stratégie utile pour rejoindre les personnes à risque de contracter des ITS puisque les services sont offerts à des populations qui ne sont pas normalement au courant des services ou qui ne sont pas en mesure d'y accéder en raison de leur situation sociale (16). Des évaluations antérieures des services de sensibilisation à Edmonton, notamment le recours à des mesures incitatives, ont mis en évidence l'utilité de ces services pour repérer les nouveaux cas (17).

Nous avons constaté que la gestion des cas par les infirmières accroît le lien avec les soins et améliore les effets pour les patients $(18,19)$. Dans notre population à l'étude, les patientes qui n'ont pas eu de contact avec la santé publique ont eu de moins bons résultats, car le risque d'accoucher d'un nourrisson atteint de syphilis congénitale était multiplié par 3,6. Dans l'ensemble, le temps médian entre le premier prélèvement et le début du traitement était de six jours, et seulement cinq cas 
n'avaient pas reçu de traitement. Nous supposons que notre succès de traitement est lié au rôle de gestion de cas que les INP jouent dans la mobilisation des clients et la priorisation des patientes enceintes et de leurs contacts sexuels.

\section{Limites de l'étude}

Il s'agit de la première étude canadienne décrivant les effets des nourrissons nés de patientes atteintes de syphilis infectieuse pendant la grossesse. L'une des limites possibles de notre étude est que le nombre de cas signalés pourrait sous-estimer le nombre de patientes enceintes atteintes de syphilis, surtout en raison de la réduction des services de santé et du fait que les patientes ont choisi de ne pas avoir accès aux soins pendant la pandémie de la maladie à coronavirus de 2019, ainsi que les patientes qui n'ont pas pu être jointes par les INP. De plus, comme nous avons utilisé des données rétrospectives recueillies à des fins de surveillance, il manquait des données sur les caractéristiques comportementales, comme I'UDI et les rapports sexuels transactionnels dans une proportion importante de la population à l'étude, ce qui pourrait être lié à la stigmatisation sous-jacente et au biais de désirabilité sociale, entraînant donc une sous-estimation de l'impact de ces facteurs.

\section{Conclusion}

Notre étude montre que le dépistage précoce de la syphilis pendant la grossesse en respect avec les lignes directrices sur le dépistage prénatal et un solide programme de santé publique pour relier les patients aux soins en temps opportun est essentiel à la prévention des cas de syphilis congénitale. À mesure que les taux de syphilis augmentent et que les infections se propagent dans les régions rurales et éloignées où l'accès aux programmes de santé et aux programmes sociaux est limité, il est impératif que des ressources suffisantes pour le suivi en santé publique soient disponibles pour faciliter la participation des patients aux soins. De plus, un examen des pratiques actuelles de dépistage, combiné à une sensibilisation accrue non seulement des membres de l'équipe de soins de santé, mais aussi du grand public, pourrait être nécessaire pour réagir à l'évolution de l'épidémiologie de la syphilis, particulièrement à la prévalence accrue de la syphilis dans les jeunes populations hétérosexuelles. Enfin, nous devons continuer de collaborer avec les collectivités touchées afin d'offrir des services adaptés à la culture et à la société.

\section{Déclaration des auteurs}

J. G. - Contribution au concept du présent rapport, analyse statistique, contribution à l'acquisition de données, contribution à l'interprétation des données, révision critique de l'article pour le contenu intellectuel important et approbation de la version finale

P. S. - Contribution au concept du présent rapport, rédaction de la version préliminaire, contribution à l'interprétation des données, révision critique du contenu intellectuel important de l'article et approbation de la version finale
J. K. - Contribution à l'acquisition de données

L. E., L. B., A. E. S., A. C. - Contribution à l'interprétation des données, révision critique du contenu intellectuel important de l'article et approbation de la version finale

Le contenu de l'article et les points de vue qui y sont exprimés n'engagent que les auteurs et ne correspondent pas nécessairement ceux du gouvernement du Canada.

\section{Intérêts concurrents}

Aucun.

\section{Remerciements}

Nous tenons à souligner les efforts inlassables des membres du programme provincial des services d'ITS en Alberta.

\section{Financement}

Aucun.

\section{Références}

1. Rac MW, Revell PA, Eppes CS. Syphilis during pregnancy: a preventable threat to maternal-fetal health. Am J Obstet Gynecol 2017;216(4):352-63. DOI PubMed

2. Organisation mondiale de la Santé. Stratégie mondiale du secteur de la santé contre les infections sexuellement transmissibles 2016-2021. Genève (CH) : OMS; 2016. https://www.who.int/reproductivehealth/publications/rtis/ ghss-stis/fr/

3. Korenromp EL, Rowley J, Alonso M, Mello MB, Wijesooriya NS, Mahiané SG, Ishikawa N, Le LV, Newman-Owiredu M, Nagelkerke N, Newman L, Kamb M, Broutet N, Taylor MM. Global burden of maternal and congenital syphilis and associated adverse birth outcomes-Estimates for 2016 and progress since 2012. PLoS One 2019;14(2):e0211720. DOI PubMed

4. Government of Alberta. Interactive Health Data Application. Edmonton (AB): Government of Alberta; 2021. http://www.ahw.gov.ab.ca/IHDA_Retrieval/redirectToURL. do?cat $=81 \&$ subCat $=466$

5. Government of Alberta. Alberta Prenatal Screening Guidelines for Select Communicable Diseases. Edmonton (AB): Government of Alberta; 2018. https://open.alberta.ca/dataset/0ac7acb6-dc90-41338f63-5946d4bbf4d1/resource/782751ed-17b9-4116-9aa4227e55ec0299/download/alberta-prenatal-screeningguidelines-2018-10.pdf 
6. Government of Alberta. Treatment Guidelines for Sexually Transmitted Infections (STI) in Adolescents and Adults, 2018. Edmonton (AB): Government of Alberta; 2018. https://open.alberta.ca/dataset/93a97f17-5210-487da9ae-a074c66ad678/resource/bc78159b-9cc4-454e8 dcd-cc85e0fcc435/download/sti-treatment-guidelinesalberta-2018.pdf

7. Government of Alberta. Alberta Health Public Health Notifiable Disease Management Guidelines Syphilis. Edmonton (AB): Government of Alberta; 2021. https://open. alberta.ca/publications/syphilis

8. Agence de la santé publique du Canada. Syphilis infectieuse et syphilis congénitale au Canada, 2020 (infographie). Ottawa (ON) : ASPC; (modifié 2021; accédé 2022-01-22). https://www.canada.ca/fr/sante-publique/services/ publications/maladies-et-affections/syphilis-infectieusesyphilis-congenitale-canada-2020.html

9. Centers for Disease Control and Prevention. Congenital Syphilis: Preliminary 2020 Data (accédé 2022-01-21). https:// www.cdc.gov/std/statistics/2020/Congenital-SyphilispreliminaryData.htm

10. Wan Z, Zhang H, Xu H, Hu Y, Tan C, Tao Y. Maternal syphilis treatment and pregnancy outcomes: a retrospective study in Jiangxi Province, China. BMC Pregnancy Childbirth 2020;20(1):648. DOI PubMed

11. Qin JB, Feng TJ, Yang TB, Hong FC, Lan LN, Zhang CL, Liu XL, Yang YZ, Xiao SY, Tan HZ. Synthesized prevention and control of one decade for mother-to-child transmission of syphilis and determinants associated with congenital syphilis and adverse pregnancy outcomes in Shenzhen, South China. Eur J Clin Microbiol Infect Dis 2014;33(12):2183-98. DOI PubMed

12. Hersh AR, Megli CJ, Caughey AB. Repeat Screening for Syphilis in the Third Trimester of Pregnancy: A Cost-Effectiveness Analysis. Obstet Gynecol 2018;132(3):699-707. DOI PubMed

13. Plitt SS, Osman M, Sahni V, Lee BE, Charlton C, Simmonds K. Examination of a prenatal syphilis screening program, Alberta, Canada: 2010-2011. Can J Public Health 2016;107(3):e285-90. DOI PubMed
14. Agence de la santé publique du Canada. Guide sur la Syphilis: Informations importantes et ressources. Ottawa (ON) : ASPC; 2020. https://www.canada.ca/fr/santepublique/services/maladies-infectieuses/sante-sexuelleinfections-transmissibles-sexuellement/lignes-directricescanadiennes/syphilis.html

15. Workowski KA, Bachmann LH, Chan PA, Johnston CM, Muzny CA, Park I, Reno H, Zenilman JM, Bolan GA. Sexually Transmitted Infections Treatment Guidelines, 2021. MMWR Recomm Rep 2021;70(4):1-187. DOl PubMed

16. National Collaborating Centre for Infectious Diseases. Outreach with Vulnerable Populations. Winnipeg (MB): NCCID; 2011. https://nccid.ca/publications/outreach-withvulnerable-populations/

17. Gratrix J, Fan S, Anderson B, Conroy P, Hewitt S, Parnell T, Jaipual J, Parker P, Singh AE, Talbot J. The Use of Financial Compensation as an Incentive for Infectious Syphilis Case Finding among Vulnerable Populations in Edmonton, Canada. Proceedings of the 19th Biennial Conference of the International Society for Sexually Transmitted Diseases Research; 2011 July 10-13; Quebec City, Canada. Sexually Transm Infect. 2011;87(Suppl 1): Poster P5-S4.05. https://sti. bmj.com/content/87/Suppl_1

18. Starbird LE, Han HR, Sulkowski MS, Budhathoki C, Reynolds NR, Farley JE. Care2Cure: A randomized controlled trial protocol for evaluating nurse case management to improve the hepatitis $C$ care continuum within HIV primary care. Res Nurs Health 2018;41(5):417-27. DOI PubMed

19. Starbird LE, Budhathoki C, Han HR, Sulkowski MS, Reynolds NR, Farley JE. Nurse case management to improve the hepatitis $\mathrm{C}$ care continuum in HIV co-infection: results of a randomized controlled trial. J Viral Hepat 2020;27(4):376-86. DOl PubMed 